
was not certified by peer review) is the author/funder. All rights reserved. No reuse allowed without permission.

\title{
If Peer Community In Evolutionary Biology
}

Sofonea MT, Aldakak L, Boullosa LFVV, Alizon S. 2017.

Can Ebola Virus evolve to be less virulent in humans?

bioRxiv 108589, http://dx.doi.org/10.1101/108589

A Preprint reviewed and recommended by Peer Community In Evolutionary Biology:

http://dx.doi.org/10.24072/pci.evolbiol.100022 


\title{
Can Ebola Virus evolve to be less virulent in humans?
}

\author{
Mircea T. Sofonea ${ }^{1, *}$, Lafi Aldakak ${ }^{1,2}$, Luis Fernando Boullosa ${ }^{1,2}$, Samuel Alizon ${ }^{1}$
}

${ }^{1}$ Laboratoire MIVEGEC (UMR CNRS 5290, IRD 224, UM) 911 avenue

5

\author{
Agropolis, B.P . 64501, 34394 Montpellier Cedex 5, France \\ ${ }^{2}$ Erasmus Mundus Master Programme in Evolutionary Biology (MEME) \\ * Author for correspondence: mircea.sofonea@normalesup.org
}

Article Type: Letter

Short Running title: Modeling Ebola virulence evolution

Keywords: case fatality ratio, virulence, EBOV, transmission, evolutionary epidemiology,

public health, trade-offs, outbreak, adaptation

Abstract Word Count: 160 words

Total Word Count: Approximately 4500 words 


\section{Abstract}

This preprint has been reviewed and recommended by Peer Community In Evolutionary Biology (<http://dx.doi.org/10.24072/pci.evolbiol.100022>). Understanding Ebola Virus (EBOV) virulence evolution is not only timely but also raises specific questions because it causes one of the most virulent human infections and it is capable of transmission after the death of its host. Using a compartmental epidemiological model that captures three transmission routes (by regular contact, via dead bodies and by sexual contact), we infer the evolutionary dynamics of case fatality ratio (CFR) on the scale of an outbreak and on the long term. Our major finding is that the virus's specific life cycle imposes selection for high levels of virulence and that this pattern is robust to parameter variations in biological ranges. In addition to shedding a new light on the ultimate causes of EBOV's high virulence, these results generate testable predictions and contribute to informing public health policies. In particular, burial management stands out as the most appropriate intervention since it decreases the $\mathcal{R}_{0}$ of the epidemics, while imposing selection for less virulent strains. 


\section{Impact Summary}

The severe haemorrhagic fever caused by Ebola Virus (EBOV) usually kills more than one infected individual out of two in the absence of treatment, which makes this pathogen one of the most virulent known to humans. The recent outbreak in West Africa (2013-2016) revealed that the virus is able to spread and persist for months across countries. It is often thought that virulence could be due to the fact that the virus is adapted to its reservoir host. Given that microbes evolve rapidly, it is important to determine whether EBOV virulence is likely to decrease as the virus adapts to its human host. To address this problem, we developed a novel mathematical model tailored to EBOV's life cycle, notably by capturing its three main transmission routes (by regular contact, sexual contact and via dead bodies). We investigated the evolutionary trends of EBOV's virulence on different time scales (outbreak initiation, short term and long term). Our results reveal that the virulence of EBOV might not be due to the maladaptation of the virus, but could rather originate from its unique life cycle. These results are robust to the parameter values chosen.

From a public health perspective, burial management stands out as the main leverage to fight the virulence of EBOV, both on the short and long terms. 


\section{Introduction}

Ebola Virus (EBOV) has been a major source of fear since its discovery in 1976. Until 2013, all outbreaks could be traced to spillover from reservoir hosts (Leroy et al. 2005) and were limited in size. This was attributed to EBOV's extremely high case fatality ratio (CFR), that is the ratio of infected hosts who die from the infection, which we use here as a measure of infection virulence. The dramatic 2013-2016 epidemic in West Africa, which caused more than 28,000 infections and claimed at least 12,000 lives, showed that the virus can persist in the human population for months, therefore raising the question: 'How will the virulence of Ebola Virus evolve in humans?' (Kupferschmidt, 2014).

Being an RNA virus, Ebola is prone to rapid evolution (de La Vega et al., 2015) and in vitro analyses suggest that the virus has evolved during the outbreak towards an increased tropism for human cells (Urbanowicz et al. 2016). It was first thought that host-parasite interactions should always evolve towards benignity because mild strains seem to have a transmission advantage over strains that kill their hosts rapidly (Méthot, 2012). Since the 1980s, evolutionary biologists have shown that parasite virulence can be adaptive because it may correlate with transmissibility or within-host competitiveness (for a review, see Alizon and Michalakis, 2015). The avirulence theory does remain prevalent in many fields. For instance, some envisage a decrease in EBOV virulence due to host adaptation Kupferschmidt (2014), even though we know the virulence of

some human infectious diseases such as HIV or tuberculosis has followed an increasing trend since their emergence (Gagneux, 2012, Herbeck et al., 2012).

Studying virulence as a potentially adaptive trait for the parasite requires encompassing the whole epidemiological life cycle of the infectious agent (Alizon and Michalakis, 2015). In the case of Ebola Virus, most individuals acquire the infection after direct contact with blood, bodily 

mortem transmission route cannot be neglected in Ebola Virus epidemics (Chan, 2014), although its magnitude is likely to be low compared to direct transmission (Weitz and Dushoff, 2015). From an evolutionary standpoint, this route might be crucial as well since the timing of life-history events can dramatically affect virulence evolution (Day, 2003). Intuitively, if the virus is still potential. Moreover, there is an increasing evidence that EBOV might also be transmitted through sexual contact even long after the clinical 'clearance' of the infection since the virus can persist in the semen for months (Eggo et al., 2015; Thorson et al., 2016; Uyeki et al., 2016).

Will EBOV become more virulent by adapting to humans? To address this question, we use 75 over more virulent ones. 


\section{Methods}

For clarity, most of the technical details are shown in Supplementary materials and this section values.

\section{Epidemiological model}

Our original compartmental model is based on the classical Susceptible-Exposed-Infected-Recovered $(S E I R)$ model, which we enhanced by adding a convalescent class $(C)$ that allows for sexual trans-

mission (Abbate et al. 2016) and an infected dead body class (D) that allows for post-mortem transmission (Legrand et al., 2007; Weitz and Dushoff, 2015). The model is deterministic and does not include additional host heterogeneity, spatial structure or public health interventions.

We incorporated demography through a host inflow term $\lambda$ and a baseline mortality rate $\mu$. Susceptible individuals $(S)$ can become infected by regular contact with symptomatic infected individuals $(I)$ (World Health Organization Ebola Response Team, 2014), by sexual contact with convalescent individuals $(C)$ (Mate et al., 2015) and by contact with the dead body of EVD victims, mostly during ritual practices $(D)$ (Chan, 2014). The rates at which these events occur are proportional to $\beta_{I}, \beta_{C}$ and $\beta_{D}$ respectively. As in most models (Keeling and Rohani, 2008), we assumed sexual transmission to be frequency-dependent. For non-sexual transmission, we assumed density-dependent transmission following an analysis of the 2013-2016 epidemic (Leander et al. 2016), although performed at a smaller scale than ours. The total population size of live hosts is denoted $N$.

Upon infection, susceptibles move to the so-called 'exposed' class $(E)$, meaning they are infected but not yet infectious. For Ebola Virus infections, this latency period is also the incubation period, 


$$
\text { rate } \omega \text {. }
$$

At the end of this latency/incubation period, individual move to the symptomatic infected compartment $(I)$. They leave this compartment at a rate $\gamma$, which we calibrated using the average time elapsed from the onset of the symptoms to death or recovery. We hereafter refer to this as the 115 The case fatality ratio (CFR), $\alpha$, is our measure of virulence.

We assumed that infected individuals who survive the infection clear the virus from their bloodstream but not from other fluids such as semen and may therefore still transmit EBOV through sexual contacts (Deen et al. 2015). These convalescent individuals $(C)$ completely eliminate EBOV we strongly vary this parameter since it represents an important leverage public health policies have on the epidemics. In the absence of burial team intervention, body fluids from dead bodies remain infectious for a period $\varepsilon^{-1}$ which is known to be less than 7 days in macaques Prescott et 
al. 2015).

Our model, pictured in Figure 1, consists in a set of Ordinary Differential Equations (ODEs) shown in Supplementary Material A,

Table 1 lists the 11 model parameters. Their values were calibrated using data from the 20132016 epidemic in West Africa. We worked at a country level and preferentially chose estimates from the Liberia outbreak, because with approximately 10,000 cumulative cases (World Health

Organization, 2016), its magnitude lies in between that of Sierra Leone and Guinea. Demography data from Liberia were obtained from publicly available data from the Central Intelligence Agency (Central Intelligence Agency, 2016). The newborn inflow was set such that the disease free equilibrium matches the country's population size.

In Supplementary Material C, we calculate the basic reproduction number of EBOV (denoted $\mathcal{R}_{0}$ ), which is the average number of secondary infections caused by a single infected individual in a fully susceptible population (Diekmann et al. 1990). By studying the local stability of the system (S1) at the disease free equilibrium, we found that

$$
\mathcal{R}_{0}=\frac{\beta_{I}}{\gamma} S_{0}+\frac{\alpha \theta \beta_{D}}{\varepsilon} S_{0}+\frac{(1-\alpha) \beta_{C}}{\sigma},
$$

where $S_{0}=\lambda / \mu$ is the total population size before the epidemic. The three terms in $\mathcal{R}_{0}$ correspond to each transmission route: from symptomatic individuals $\left(\mathcal{R}_{0, I}:=\beta_{I} S_{0} / \gamma\right)$, infectious bodies $\left(\mathcal{R}_{0, D}:=\alpha \theta \beta_{D} S_{0} / \varepsilon\right)$, and convalescent individuals $\left(\mathcal{R}_{0, C}:=(1-\alpha) \beta_{C} / \sigma\right)$. Notice that the incubation period does not affect $\mathcal{R}_{0}$. 


\section{Transmission-virulence trade-off and estimated values}

Trade-offs are a central component of evolutionary model and, without them, predictions tend to be trivial (e.g. viruses should evolve to maximise their transmission rates and minimise their

et al., 2015) but demonstrating a clear link is challenging (for HIV, it has required identifying sero-discordant couples in cohorts).

We assumed an increasing relationship between transmission rates and $\mathrm{CFR}(\alpha)$ such that:

$$
\beta_{H}(\alpha):=b_{H} \alpha^{p}
$$

where $b_{H}$ is a constant factor, $p \in \mathbb{R}_{+}$is a parameter capturing the concavity of the trade-off curve and $H$ stands for one of the compartment $I, D$ or $C$. The exact value of $p$ results from within-host interactions (Alizon and van Baalen, 2005) but one can identify four kinds of trade-offs: $p>1$ corresponds to an amplifying transmission benefit from increasing $\mathrm{CFR}, p=1$ corresponds 
to a linear relationship between transmission and CFR, $0<p<1$ corresponds to a saturating transmission benefit from increasing CFR and $p=0$ is the degenerate case without trade-off. From a biological standpoint, we could expect different transmission routes to have different trade-off shapes $(p)$ but, as we show here, our results are largely independent of $p$.

Transmission rates being difficult to estimate (Leander et al., 2016), we indirectly infer the order of magnitudes of $b_{I}, b_{C}$ and $b_{D}$ by setting the $\mathcal{R}_{0}$ close to 2 , that is its approximate value for the 2014 epidemic (World Health Organization Ebola Response Team, 2014). Since $\mathcal{R}_{0}$ is the sum of the epidemiological contributions of each transmission route (see equation (1)), we added

the constrain that, according to previous studies, transmission from symptomatic individuals contributes about ten times more than transmission from dead bodies (World Health Organization Ebola Response Team, 2014, Weitz and Dushoff, 2015) and one hundred times more than transmission from convalescent individuals (Abbate et al. 2016). Straightforward calculations (analogous to those done for sensitivity analysis in Supplementary Material E) resulted in attributing the orders of magnitude shown in Table 1 .

In the following, the exponent $p$ was left undetermined, but its value was set to 0 for the estimation of $b_{H}$ in the null hypothesis. This leads to $\mathcal{R}_{0} \approx 1.86$, which is very close to the ¿WHO mean estimation for the Liberia epidemic, namely 1.83 (World Health Organization Ebola Response Team, 2014).

\section{Long term evolution}

We used the adaptive dynamics framework (Geritz et al., 1998), which assumes that evolution proceeds by rare and small phenotypical mutations occurring in a monomorphic population that has reached ecological stationarity. Polymorphism is therefore limited to transient dimorphic phases where the ancestor (hereafter called the 'resident') and its mutant compete. Depending on 
Supplementary Material C.3p to the mutant relative reproduction number

$$
\mathcal{R}\left(\alpha^{\prime}, \alpha\right)=\frac{\frac{\beta_{I}\left(\alpha^{\prime}\right)}{\gamma}+\frac{x \theta \beta_{D}\left(\alpha^{\prime}\right)}{\varepsilon}+\frac{\left(1-\alpha^{\prime}\right) \beta_{C}\left(\alpha^{\prime}\right)}{\sigma \widetilde{N}(\alpha)}}{\frac{\beta_{I}(\alpha)}{\gamma}+\frac{\alpha \theta \beta_{D}(\alpha)}{\varepsilon}+\frac{(1-\alpha) \beta_{C}(\alpha)}{\sigma \widetilde{N}(\alpha)}},
$$

where there total host population size can be approximated (see Supplementary Material B) by

$$
\widetilde{N}(\alpha) \approx(1-\alpha) S_{0}+\frac{\alpha}{\frac{\beta_{I}(\alpha)}{\gamma}+\frac{\alpha \theta \beta_{D}(\alpha)}{\varepsilon}}
$$

We then calculated the selection gradient by deriving the relative reproduction number (equation (3p) with respect to the mutant's trait (Otto and Day, 2007). Equating the mutant and resident trait value we eventually found

$$
\Delta(\alpha)=\frac{p}{\alpha}+\frac{\frac{\theta b_{D}}{\varepsilon} \widetilde{N}(\alpha)-\frac{b_{C}}{\sigma}}{(1-\alpha) \frac{b_{C}}{\sigma}+\left(\frac{b_{I}}{\gamma}+\frac{\theta b_{D}}{\varepsilon} \alpha\right) \widetilde{N}(\alpha)}
$$


The sign of $\Delta$ indicates the way natural selection acts on the trait depending on the resident's trait.

\section{Short term evolution}

Viruses evolve so rapidly that evolutionary and epidemiological dynamics may overlap. The epito get a broader picture. 


\section{Results}

\section{Virulence and emergence}

If we focus on the lowest virulence that may lead to an epidemic (denoted $\alpha_{\min }$ ), we find that with our default parameter values burial management can prevent emergence (that is bring $\mathcal{R}_{0}$ below unity by moving vertically in Figure 2 only if the transmission-virulence trade-off is strong enough (the green, blue and cyan curves).

In the following, we will generally assume that EBOV is adapted enough to persist in the human population $\left(\mathcal{R}_{0}>1\right)$. Since outbreak originates via spillover from reservoir hosts (Leroy et al. 2000), it is likely that the virus is maladapted in the first human infections. However, to capture these dynamics, an evolutionary rescue formalism would be more appropriate given the importance of stochastic events and this is outside the scope of this study (for a review, see Gandon et al., 2013).

\section{Long-term virulence evolution}

If the selection gradient in equation (5) is negative for any $\operatorname{CFR}(\Delta(\alpha)<0)$, then the virus population evolves towards its lowest virulence that allows persistence (that is $\alpha_{\min }$ ). If the gradient 

only be reached if there exists $\alpha^{\star}$ such that $\Delta(\alpha) \geq 0$ for $\alpha \leq \alpha^{\star}, \Delta(\alpha) \leq 0$ for $\alpha \geq \alpha^{\star}$ and $\mathcal{R}\left(\alpha, \alpha^{\star}\right)<1$ for any $\alpha \neq \alpha^{\star}$. We show in Supplementary Material D.4 that this occurs only if the proportion of unsafe burials $(\theta)$ and the trade-off parameter $(p)$ are lower than the following boundaries

$$
\theta<\frac{b_{I} b_{C} \varepsilon}{\gamma \sigma b_{D}} \text { and } p<\frac{b_{C}}{\sigma}
$$

Unless these two conditions are met, the selection gradient is always positive and EBOV is expected to always evolve towards higher case fatality ratios $\left(\alpha^{\star}=1\right)$. Rewriting the first inequality as $\frac{\theta b_{D} S_{0}}{\varepsilon}<\frac{b_{I} S_{0}}{\gamma} \times \frac{b_{C}}{\sigma}$ highlights that virulence is favoured by natural selection as soon as the post mortem transmission component is greater than the product of the symptomatic and convalescent transmission components.

Figure 3 shows how $\alpha^{\star}$ is numerically affected by a change in burial management $(\theta)$ and trade-off strength $(p)$. Unless the proportion of safe burials is brought below 4\%, and unless there is a weak trade-off $(p<0.01)$, CFR will remain high. Intuitively, this double condition can be understood in the following way. If the trade-off is negligible, the CFR is weakly linked to transmission by regular contact and therefore selection on $\alpha$ only weakly depends on this component of the life cycle. As a consequence, the value of $\theta$ governs the relative importance of the two transmission routes that matter. Post mortem transmission always favours higher CFR, whereas the sexual transmission route can be maximised for intermediate levels of virulence (see Supplementary Material $\mathrm{H}$.)

It was not possible to find an explicit expression for the long-term equilibrium virulence $\left(\alpha^{\star}\right)$, 
but we found it lies in the following interval (Supplementary Material D.5):

$$
\alpha^{\star} \in\left[\frac{p}{\frac{b_{C}}{\sigma}-\frac{\theta \frac{b_{D}}{\varepsilon}}{\frac{b_{I}}{\gamma}+\theta \frac{b_{D}}{\varepsilon}}}, \frac{\left(\left(\frac{b_{I}}{\gamma}+\theta \frac{b_{D}}{\varepsilon}\right) S_{0}+\frac{b_{C}}{\sigma}\right) p}{(1+p) \frac{b_{C}}{\sigma}-\theta \frac{b_{D}}{\varepsilon} S_{0}}\right] .
$$

The lower bound of this interval increases with trade-off strength $(p)$ and intensity of the post mortem transmission route $\left(\theta b_{D} / \varepsilon\right)$. If post mortem transmission is strongly reduced, owing to a safer burial management $(\theta \rightarrow 0)$, the lower bound simplifies to $p \sigma / b_{C}$. The long-term virulence then appears to be a balance between trade-off strength and sexual transmission intensity, which is consistent with our intuitive explanation of the condition for an intermediate virulence to be selected. In particular, any decrease in the time for convalescent hosts to clear the virus (i.e. increase in $\sigma$ ) will increase the lower bound for CFR.

To further assess the robustness of these results, we performed a sensitivity analysis by varying the relative importance of each transmission route (regular contact, sexual transmission and

transmission from dead bodies), while keeping the total value of $\mathcal{R}_{0}$ constant. As shown in Figure 4. unless the values of $p$ are extremely low, variations in the relative transmission routes is unlikely to be sufficient to move our default value (dotted lines) to the region where low virulences (e.g. $\alpha<50 \%$ ) are favored (green area). To give a quantitative estimate, the relative importance of transmission via sexual contact (on the vertical axis) would need to be about 40 times greater than the current estimates to bring the current estimate above the blue separatrix, which already assumes a low trade-off and a perfect burial management.

\section{Short term evolutionary dynamics}

Reaching an evolutionary equilibrium may take time (especially if strains have similar trait values) and the transient dynamics can be non-trivial because the system is non-linear. The Price equation 

considering the initial diversity in the virus population.

If we denote by $\bar{x}^{H}$ the average value of trait $x$ in compartment $H$ and by $\operatorname{cov}_{H}(x, y)$ the statistical covariance between traits $x$ and $y$ in compartment $H$ (which becomes the statistical variance $\operatorname{var}_{H}(x)$ if $\left.x \equiv y\right)$, the dynamics of average virulence in the four infected compartments satisfy the following ODEs (see Supplementary Material F for further details):

$$
\begin{aligned}
\frac{\mathrm{d} \bar{\alpha}^{I}}{\mathrm{~d} t} & =-\operatorname{cov}_{I}(\alpha, \gamma)+\left(\operatorname{cov}_{E}(\alpha, \omega)+\left(\bar{\alpha}^{E}-\bar{\alpha}^{I}\right) \bar{\omega}^{E}\right) \frac{E_{\bullet}}{I_{\bullet}} \\
\frac{\mathrm{d} \bar{\alpha}^{E}}{\mathrm{~d} t} & =-\operatorname{cov}_{E}(\alpha, \omega)+\frac{S}{E_{\bullet}} \sum_{H \in\{I, D, C\}}\left(\operatorname{cov}_{H}\left(\beta_{H}, \alpha\right)+\left(\bar{\alpha}^{H}-\bar{\alpha}^{E}\right) \bar{\beta}_{H}^{H}\right) H_{\bullet} \\
\frac{\mathrm{d} \bar{\alpha}^{D}}{\mathrm{~d} t} & =-\operatorname{cov}_{D}(\alpha, \varepsilon)+\left(\operatorname{var}_{I}(\alpha)+\bar{\alpha}^{I}\left(\bar{\alpha}^{I}-\bar{\alpha}^{D}\right)\right) \theta \gamma \frac{I_{\bullet}}{D_{\bullet}} \\
\frac{\mathrm{d} \bar{\alpha}^{C}}{\mathrm{~d} t} & =-\operatorname{cov}_{C}(\alpha, \sigma)+\left(\operatorname{cov}_{I}(\alpha, \gamma)-\gamma \operatorname{var}_{I}(\alpha)+\left(1-\bar{\alpha}^{I}\right)\left(\bar{\alpha}^{I}-\bar{\alpha}^{C}\right) \bar{\gamma}^{I}\right) \frac{I_{\bullet}}{C_{\bullet}}
\end{aligned}
$$

where $H_{\bullet}:=\sum_{i=1}^{n} H_{i}$ denotes the total size of compartment $H \equiv E, I, D, C$.

Focusing on the compartment on which virulence acts, namely the symptomatic individuals, indicates that the short-term evolution of the average virulence $\bar{\alpha}^{I}$ is mainly governed by the correlations between this trait and the symptomatic and latency periods. More explicitly, equation (8a) states that if the most virulent strains induce the longest symptomatic period and/or the shortest latency periods, the average virulence in $I$ can be expected to increase at the beginning of the epidemic. Intuitively, newly symptomatic individuals are more likely to have been infected with a highly virulent strain.

Equation 8a contains a third term proportional to $\bar{\alpha}^{E}-\bar{\alpha}^{I}$, which is more difficult to apprehend. Indeed, $\bar{\alpha}^{E}$ varies as well and follows a complicated ODE that involves not only the correlation with the latency period but also correlations with the transmission rates (equation (8b)). 
This diversity of components make both $\bar{\alpha}^{E}$ and $\bar{\alpha}^{I}$ difficult to predict early in the epidemics.

We therefore simulated epidemics numerically according to system (S2). We considered nine

\section{Discussion}

\section{Virulence could be adaptive for EBOV}

Ebola Virus is one of the deadliest human pathogen (Feldmann and Geisbert, 2011). The recent epidemic in West Africa has shown that it can transmit for months months among humans throughout entire countries. As any microbe (especially RNA viruses), it is likely exposed to fast 

because it cannot directly be addressed by public health policies. Finally, even if these conditions are met, the level of virulence reached in the long term may still be high, especially if sexual transmission is limited. On a more positive note, results from the Price equation approach show 
that the virus may experience transitory lower levels of virulence before reaching this maximum

In addition to the strong selection for maximum virulence of EBOV, another striking result is that decreasing the ratio of unsafe burials is triply effective. First, it decreases the spread of the virus (i.e. its $\mathcal{R}_{0}$ ). Second, in the short term, it can help limit a transitory increase in virulence. Indeed, in the first weeks of an epidemic, the sexual transmission route is negligible compared to the other routes that are maximised for maximum CFR. Third, in the long term, decreasing the proportion of unsafe burials is necessary to shift the selective pressure in favour of less virulent strains.

\section{Virulence is a shared trait}

In evolutionary biology, virulence is defined as the decrease in host fitness due to the infection (Alizon and Michalakis, 2015). Given the speed at which a pathogen kills its host, EBOV's virulence can neither be measured as a decrease in instantaneous fecundity (which would be almost zero) nor as an increase of instantaneous mortality rate (which would tend towards infinity or zero

depending on the infection outcome). The case fatality ratio (CFR) therefore appears to be the only measurable and epidemiologically relevant proxy for EBOV's virulence.

We focused on the virus side but, like any infection trait, virulence is also determined by the host and its environment. To predict how virulence will change in the future, we should also consider how hosts may change. In the case of EBOV, it was known before the recent epidemics that some people can become immune to the virus without exhibiting any symptoms (Leroy et al. 2000). The question remains to know if they can also be infectious. More generally, our assumption 
of life-long protection could be oversimplifying.

Finally, to make predictions on the long term evolution, we need to factor in how the virus population will evolve in response to the variation in the host population's immune status. Since can create epidemiological feedbacks that alter this prediction by causing virulence to be maximised for intermediate levels of population structures (Lion and Boots, 2010). This is why predicting virulence evolution in a fully spatially structured model is beyond the scope of this study. 


\section{Testable predictions}

400

valuable. Note that in the case of EBOV, it is not the exact shape that matters but rather the general trend. 


\section{Conclusion}

420 emergence, short-term or long-term dynamics, can be explained by its particular life cycle that mixes parasitism and parasitoidism (post mortem transmission). Unfortunately, any long term decrease in virulence is unlikely for West African strains at any time scale, although increasing the safe burial proportion appears to be an optimal response in both the short and long terms.

${ }_{425}$ Acknowledgements

\section{Funding Statement}

M. T. Sofonea and S. Alizon acknowledge support from the CNRS, the UM and the IRD.

\section{Competing Interests}

We have no competing interests.

\section{${ }_{430}$ Authors' Contributions}

Conceived the project: all

Performed the analysis: MS

Wrote the article: all 


\section{References}

Abbate, J. L., C. L. Murall, H. Richner and C. L. Althaus. 2016. Potential Impact of Sexual Transmission on Ebola Virus Epidemiology: Sierra Leone as a Case Study. PLoS Negl Trop Dis $10(5): 1-15$.

Ali, H., B. Dumbuya, M. Hynie, P. Idahosa, R. Keil and P. Perkins. (2016). The Social and Political Dimensions of the Ebola Response: Global Inequality, Climate Change, and Infectious Disease, Pages 151-169 in W. Leal Filho, M. U. Azeiteiro and F. Alves, eds. Climate Change and Health: Improving Resilience and Reducing Risks, Springer International Publishing, Switzerland, pp. 151-169.

Alizon, S. and M. van Baalen. 2005. Emergence of a convex trade-off between transmission and virulence. Am. Nat. 165(6):E155-E167.

Alizon, S. and Y. Michalakis. 2015. Adaptive virulence evolution: the good old fitness-based approach. Trends Ecol Evol 30(5):248-254.

Alizon, S., V. von Wyl, T. Stadler, R. D. Kouyos, S. Yerly, B. Hirschel, J. Böni, C. Shah, T. Klimkait, H. Furrer, A. Rauch, P. Vernazza, E. Bernasconi, M. Battegay, P. Bürgisser, A. Telenti, H. F. Günthard, S. Bonhoeffer and the Swiss HIV Cohort Study. 2010. Phylogenetic approach reveals that virus genotype largely determines HIV set-point viral load. PLoS Pathog. 6(9):e1001123.

Anderson, R. M. and R. M. May. 1981. The Population Dynamics of Microparasites and Their Invertebrate Hosts. Phil. Trans. R. Soc. Lond. B 291(1054):451-524.

Bausch, D. G., J. S. Towner, S. F. Dowell, F. Kaducu, M. Lukwiya, A. Sanchez, S. T. Nichol, 
T. G. Ksiazek and P. E. Rollin. 2007. Assessment of the risk of Ebola virus transmission from bodily fluids and fomites. J. Infect. Dis. 196(S2):S142-S147.

Berngruber, T. W., R. Froissart, M. Choisy and S. Gandon. 2013. Evolution of virulence in emerging epidemics. PLoS Pathog. 9(3):e1003209.

Boots, M. and A. Sasaki. 1999. 'Small worlds' and the evolution of virulence: infection occurs $460 \quad$ locally and at a distance. Proc. R. Soc. Lond. B 266:1933-1938.

Central Intelligence Agency (2016). The World Factbook.

Chan, M. 2014. Ebola Virus Disease in West Africa - No Early End to the Outbreak. N. Engl. J. Med. 371(13):1183-1185.

Cousineau, S. V. and S. Alizon. 2014. Parasite evolution in response to sex-based host heterogeneity in resistance and tolerance. J Evol Biol 27(12):2753-66.

Crowe, S. J., M. J. Maenner, S. Kuah, B. R. Erickson, M. Coffee, B. Knust, J. Klena, J. Foday, D. Hertz, V. Hermans et al. 2016. Prognostic indicators for Ebola patient survival. Emerg. Infect. Dis. 22(2):217.

Day, T. 2003. Virulence evolution and the timing of disease life-history events. Trends Ecol. Evol. 18(3):113-118.

Day, T. and S. Gandon. (2006). Insights from Price's equation into evolutionary epidemiology, Pages 23-44 in Z. Feng, U. Dieckmann and S. A. Levin, eds. Disease Evolution: Models, Concepts, and Data Analyses, Vol. 71 of DIMACS Series in Discrete Mathematics and Theoretical Computer Science, American Mathematical Society, Providence, RI (USA), pp. 23-44. 
475

Day, T. and S. R. Proulx. 2004a. A general theory for the evolutionary dynamics of virulence. Am. Nat. 163(4):E40-E63.

Day, T. and S. R. Proulx. 2004b. A general theory for the evolutionary dynamics of virulence. Am. Nat. 163(4):E40-E63.

de La Vega, M.-A., D. Stein and G. P. Kobinger. 2015. Ebolavirus evolution: past and present. PLoS Pathog 11(11):e1005221.

Deen, G. F., B. Knust, N. Broutet, F. R. Sesay, P. Formenty, C. Ross, A. E. Thorson, T. A. Massaquoi, J. E. Marrinan, E. Ervin et al. 2015. Ebola RNA persistence in semen of Ebola virus disease survivors - preliminary report. N. Engl. J. Med. PMID: 26465681.

Dieckmann, U. 2002. Adaptive dynamics of pathogen-host interactions. Pages 39-59 in U. Dieckmann, J. A. J. Metz, M. W. Sabelis and K. Sigmund, eds. Adaptive dynamics of infectious diseases: In pursuit of virulence management. Cambridge University Press.

Dieckmann, U., Metz, J. A. J., Sabelis, M. W. and Sigmund, K., eds 2002. Adaptive dynamics of infectious diseases. In pursuit of virulence management. Cambridge studies in adaptive dynamics, Cambridge University Press, Cambridge, UK.

Diekmann, O., J. Heesterbeek and J. A. Metz. 1990. On the definition and the computation of the basic reproduction ratio $R_{0}$ in models for infectious diseases in heterogeneous populations. J. Math. Biol. 28(4):365-382.

Eggo, R., C. Watson, A. Camacho, A. Kucharski, S. Funk and W. Edmunds. 2015. Duration of Ebola virus RNA persistence in semen of survivors: population-level estimates and projections. 
Feldmann, H. and T. W. Geisbert. 2011. Ebola haemorrhagic fever. Lancet 377(9768):849-862.

Fraser, C., K. Lythgoe, G. E. Leventhal, G. Shirreff, T. D. Hollingsworth, S. Alizon and S. Bonhoeffer. 2014. Virulence and pathogenesis of HIV-1 infection: an evolutionary perspective. Science $343(6177): 1243727$.

Gagneux, S. 2012. Host-pathogen coevolution in human tuberculosis. Philos. Trans. R. Soc. Lond. B 367(1590):850-859.

Gandon, S., M. E. Hochberg, R. D. Holt and T. Day. 2013. What limits the evolutionary emergence of pathogens? Philos. Trans. R. Soc. Lond. B 368:20120086.

Geritz, S. A. H., E. Kisdi, G. Meszena and J. A. J. Metz. 1998. Evolutionarily singular strategies and the adaptative growth and branching of the evolutionary tree. Evol. Ecol. 12:35-57.

Herbeck, J. T., V. Müller, B. S. Maust, B. Ledergerber, C. Torti, S. Di Giambenedetto, L. Gras, H. F. Günthard, L. P. Jacobson, J. I. Mullins and G. S. Gottlieb. 2012. Is the virulence of HIV changing? A meta-analysis of trends in prognostic markers of HIV disease progression and transmission. AIDS 26(2):193.

Hurford, A., D. Cownden and T. Day. 2010. Next-generation tools for evolutionary invasion analyses. J. R. Soc. Interface 7(45):561-71.

Keeling, M. J. and P. Rohani. 2008. Modeling infectious diseases in humans and animals. Princeton University Press.

Kupferschmidt, K. 2014. Imagining Ebola's next move. Science 346(6206):151-152.

Leander, R., W. Goff, C. Murphy and S. Pulido. 2016. Modelling Ebola within a community. Epidemiol. Infect. 144(11):2329-2337. 
Legrand, J., R. F. Grais, P.-Y. Boelle, A. J. Valleron and A. Flahault. 2007. Understanding the dynamics of Ebola epidemics. Epidemiol Infect 135(4):610-21.

Leroy, E. M., B. Kumulungui, X. Pourrut, P. Rouquet, A. Hassanin, P. Yaba, A. Délicat, J. T. Paweska, J.-P. Gonzalez and R. Swanepoel. 2005. Fruit bats as reservoirs of Ebola virus. Nature $438(7068): 575-576$.

Leroy, E. M., S. Baize, V. E. Volchkov, S. P. Fisher-Hoch, M.-C. Georges-Courbot, J. LansoudSoukate, M. Capron, P. Debré, A. J. Georges and J. B. McCormick. 2000. Human asymptomatic Ebola infection and strong inflammatory response. Lancet 355(9222):2210-2215.

Lion, S. and M. Boots. 2010. Are parasites 'prudent' in space? Ecol. Lett. 13(10):1245-1255.

Lion, S. and M. van Baalen. 2008. Self-structuring in spatial evolutionary ecology. Ecol. Lett. 11(3):277-295.

Mate, S. E., J. R. Kugelman, T. G. Nyenswah, J. T. Ladner, M. R. Wiley, T. Cordier-Lassalle, A. Christie, G. P. Schroth, S. M. Gross, G. J. Davies-Wayne et al. 2015. Molecular evidence of sexual transmission of Ebola virus. N. Engl. J. Med. 373(25):2448-2454.

Méthot, P.-O. 2012. Why do parasites harm their host? On the origin and legacy of Theobald Smith's 'law of declining virulence' - 1900-1980. Hist. Phil. Life Sci. 34(4):561-601.

Miller, M. R., A. White and M. Boots. 2006. The evolution of parasites in response to tolerance in their hosts: the good, the bad, and apparent commensalism. Evolution 60(5):945-56. Karsor, C. S. Wesseh, S. Sieh et al. 2016. Ebola and its control in Liberia, 2014-2015. Emerg. Infect. Dis. 22(2):169. 
Osterholm, M. T., K. A. Moore, N. S. Kelley, L. M. Brosseau, G. Wong, F. A. Murphy, C. J. Peters, J. W. LeDuc, P. K. Russell, M. Van Herp et al. 2015. Transmission of Ebola viruses: what we know and what we do not know. MBio 6(2):e00137-15.

Otto, S. P. and T. Day. 2007. A biologist's guide to mathematical modeling in ecology and evolution. Vol. 13, Princeton University Press.

Power, R. A., J. Parkhill and T. de Oliveira. 2017. Microbial genome-wide association studies: lessons from human GWAS. Nat Rev Genet 18(1):41-50.

Prescott, J., T. Bushmaker, R. Fischer, K. Miazgowicz, S. Judson and V. J. Munster. 2015. Postmortem stability of Ebola virus. Emerg. Infect. Dis. 21(5):856-9.

Sobarzo, A., D. E. Ochayon, J. J. Lutwama, S. Balinandi, O. Guttman, R. S. Marks, A. I. Kuehne, J. M. Dye, V. Yavelsky, E. C. Lewis et al. 2013. Persistent immune responses after Ebola virus infection. N. Engl. J. Med. 369(5):492-493.

Svennungsen, T. O. and E. Kisdi. 2009. Evolutionary branching of virulence in a single infection model. J. theor. Biol. 257:408-418.

Thorson, A., P. Formenty, C. Lofthouse and N. Broutet. 2016. Systematic review of the literature on viral persistence and sexual transmission from recovered Ebola survivors: evidence and recommendations. BMJ Open 6(1):e008859.

Towner, J. S., P. E. Rollin, D. G. Bausch, A. Sanchez, S. M. Crary, M. Vincent, W. F. Lee, C. F. Spiropoulou, T. G. Ksiazek, M. Lukwiya et al. 2004. Rapid diagnosis of Ebola hemorrhagic fever by reverse transcription-PCR in an outbreak setting and assessment of patient viral load as a predictor of outcome. J. Virol. 78(8):4330-4341. 
Urbanowicz, R. A., C. P. McClure, A. Sakuntabhai, A. A. Sall, G. Kobinger, M. A. Müller, E. C.

Holmes, F. A. Rey, E. Simon-Loriere and J. K. Ball. 2016. Human adaptation of Ebola virus during the West African outbreak. Cell 167(4):1079-1087.

Uyeki, T. M., A. K. Mehta, R. T. Davey Jr, A. M. Liddell, T. Wolf, P. Vetter, S. Schmiedel, T. Grünewald, M. Jacobs, J. R. Arribas et al. 2016. Clinical management of Ebola virus disease in the United States and Europe. N Engl J Med 374(7):636-646.

Weitz, J. S. and J. Dushoff. 2015. Modeling post-death transmission of Ebola: challenges for inference and opportunities for control. Sci. Rep. 5:8751.

World Health Organization (2016). WHO: Ebola situation report - 30 March 2016.

World Health Organization Ebola Response Team 2014. Ebola virus disease in West Africa-the first 9 months of the epidemic and forward projections. N Engl J Med 371(16):1481-95. year - slowing but not yet under control. N Engl J Med 2015(372):584-587. 
Table 1: Parameter list, description and default values.

See the main text for further details about the calibration of the transmission constants. Note that the sexual transmission constant is higher because it involves frequency-dependent transmission. ind stands for individuals. When used in the main text or in the appendix, these estimated values are denoted by a hat.

\begin{tabular}{|c|c|c|c|}
\hline Notation & Description & Default value & Reference or inference \\
\hline$\lambda$ & "Host inflow & $2 \cdot 10^{2}$ ind.day $^{-1}$ & $\begin{array}{l}\text { such that } \lambda / \mu \approx 4 \cdot 10^{6}, \text { (Central In- } \\
\text { telligence Agency, } 2016)\end{array}$ \\
\hline$\mu$ & $\begin{array}{l}\text { Host baseline mortality } \\
\text { rate }\end{array}$ & $4.5 \cdot 10^{-5} \mathrm{day}^{-1}$ & (Central Intelligence Agency, 2016) \\
\hline$b_{I}$ & $\begin{array}{l}\text { Regular contact trans- } \\
\text { mission (from infectious } \\
\text { hosts) factor }\end{array}$ & $10^{-7}$ ind $^{-1} \cdot$ day $^{-1}$ & $\begin{array}{l}\text { with the constrain } \mathcal{R}_{0, I} \approx 10 \mathcal{R}_{0, D} \\
\mathcal{R}_{0, I} \approx 10^{2} \mathcal{R}_{0, C} \text { and } \mathcal{R}_{0} \approx 1.8\end{array}$ \\
\hline$b_{C}$ & $\begin{array}{ll}\text { Sexual } & \text { transmission } \\
\text { (from } & \text { convalescent } \\
\text { hosts) factor }\end{array}$ & $10^{-4}$ day $^{-1}$ & $\begin{array}{l}\text { World Health Organization Ebola } \\
\text { Response Team, 2014; Weitz and } \\
\text { Dushoff, 2015; Abbate et al., 2016) }\end{array}$ \\
\hline$b_{D}$ & $\begin{array}{l}\text { Post mortem transmis- } \\
\text { sion (from dead hosts) } \\
\text { factor }\end{array}$ & $10^{-8}$ ind $^{-1} \cdot$ day $^{-1}$ & \\
\hline$\omega$ & Inverse of latency period & $10^{-1}$ day $^{-1}$ & $\begin{array}{l}\text { (World Health Organization Ebola } \\
\text { Response Team, } 2014)\end{array}$ \\
\hline$\alpha$ & Case fatality ratio & 0.7 & $\begin{array}{l}\text { (World Health Organization Ebola } \\
\text { Response Team, 2014) }\end{array}$ \\
\hline$\gamma$ & $\begin{array}{l}\text { Inverse of symptomatic } \\
\text { infectious period }\end{array}$ & 0.25 day $^{-1}$ & (Abbate et al., 2016) \\
\hline$\theta$ & Unsafe burial proportion & 0.25 & (Nyenswah et al., 2016) \\
\hline$\varepsilon$ & $\begin{array}{l}\text { Inverse of post mortem } \\
\text { infectious period }\end{array}$ & $10^{-1}$ day $^{-1}$ & $($ Prescott et al., 2015$)$ \\
\hline$\sigma$ & $\begin{array}{l}\text { Elimination rate of con- } \\
\text { valescent hosts }\end{array}$ & $10^{-2}$ day $^{-1}$ & $\begin{array}{l}\text { (Uyeki et al., 2016; } \\
2016)\end{array}$ \\
\hline
\end{tabular}



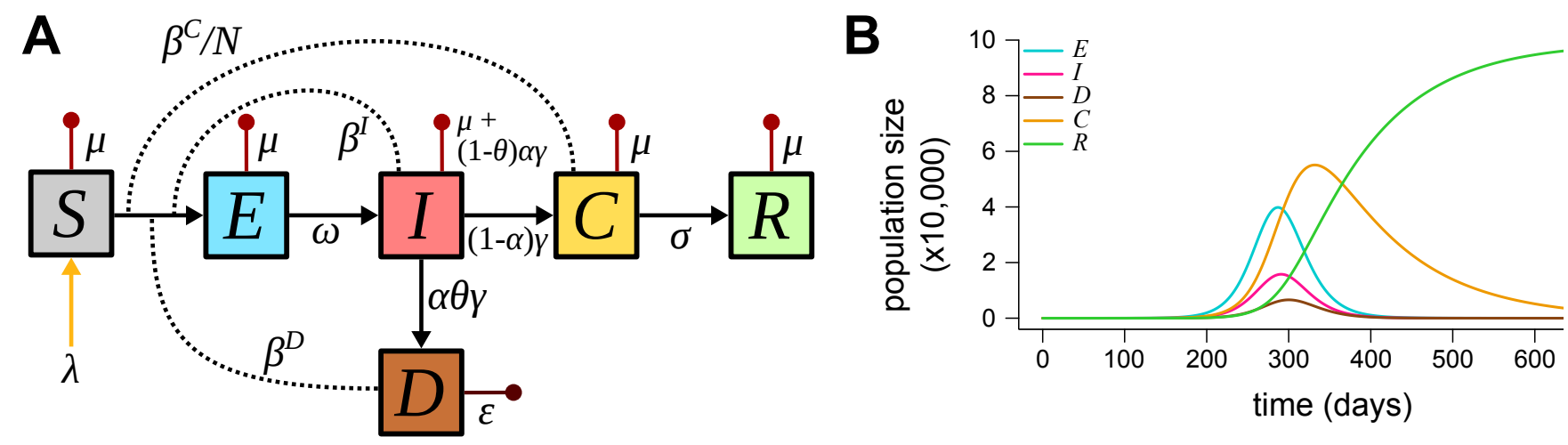

Figure 1: Epidemiology of Ebola Virus in humans. A) Epidemiological life cycle of Ebola Virus in humans and B) Population dynamics for default parameters. $S, E, I, C, R$ and $D$ are host densities that correspond to the following compartments: susceptible, exposed (infected but not yet infectious), symptomatic infected, convalescent, recovered (immunised) and dead bodies respectively. $N$ is the total living population size. Lower-case letters are rate and flow parameters, the description of which is given in Table 1 . 


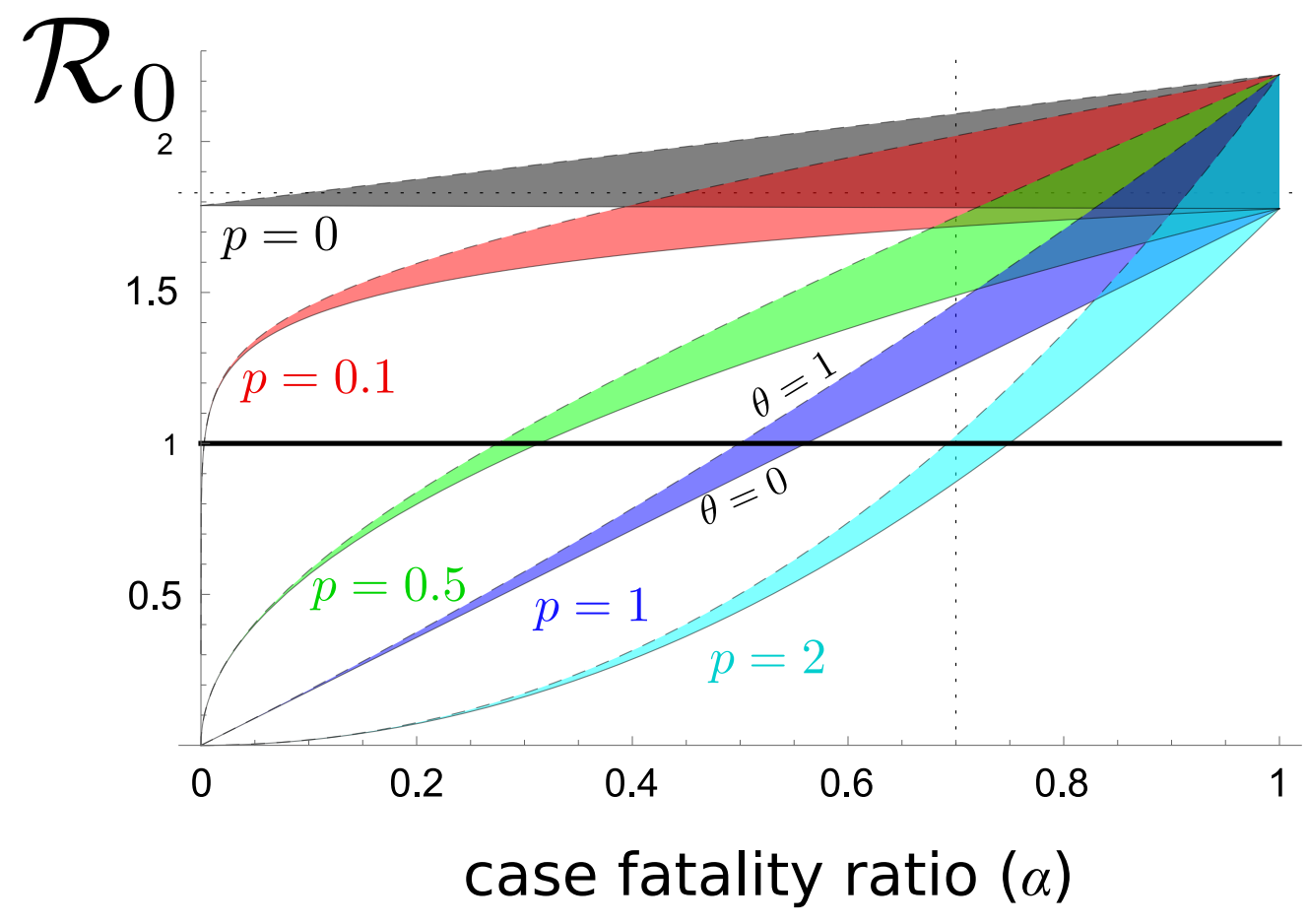

Figure 2: Basic reproduction number as a function case fatality ratio $(\alpha)$, unsafe burial ratio and trade-off shape.

Colors indicate five trade-off scenarios: absence ( $p=0$, in grey), very weak ( $p=0.1$, in red), concave ( $p=0.5$, in green), linear ( $p=1$, in dark blue), and convex $(p=2$, in light blue). The width of the coloured regions corresponds to variations in the unsafe burial ratio from completely unsafe ( $\theta=1$, dashed upper bound) to completely safe $(\theta=0$, solid lower bound). The intersection between the horizontal line and the colored areas indicates the range of $\alpha_{\min }$ for each scenario. The dotted gridlines show the $\alpha$ and $\mathcal{R}_{0}$ estimates from the literature. Other parameter values are in Table 1. See Supplementary Material D.4 for more details. 


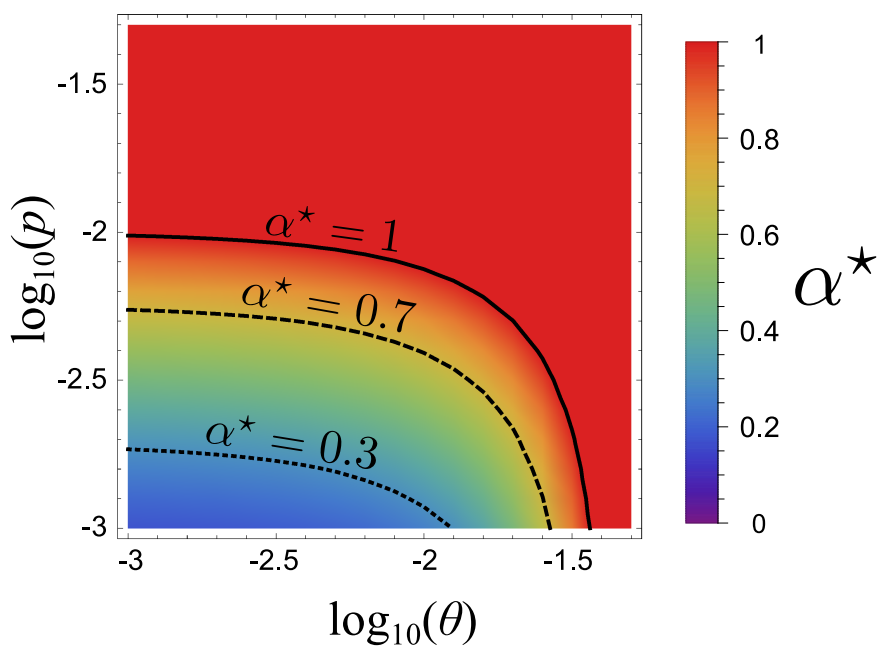

Figure 3: Evolutionary stable virulence $\left(\alpha^{\star}\right)$ as a function of unsafe burial ratio $(\theta)$ and trade-off exponent $(p)$.

The solid, dashed and dotted lines correspond to $\alpha^{\star}=1,0.7$ and 0.3 respectively. Parameter vlaues are shown in Table 1. See Supplementary Material D.4 for more details. 


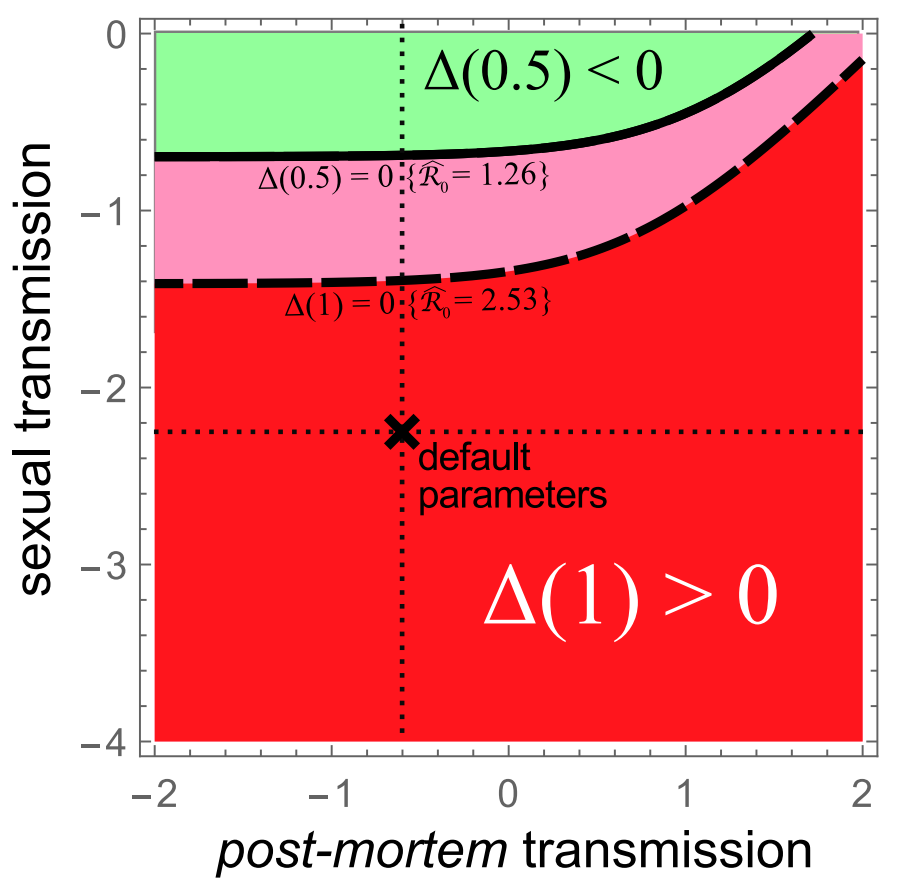

Figure 4: Sensitivity analysis of long-term virulence evolution.

The graphic shows the sign of the selection gradient for virulence when varying the relative weight (in orders of magnitude) of the post-mortem transmission component $\left(\log _{10}(\delta)\right.$, on the $x$-axis) and the sexual transmission component $\left(\log _{10}\left(\kappa / S_{0}\right)\right.$, on the $y$-axis) in the overall transmission of EBOV. When the basic reproduction number is set at its upper bound $\left(\widehat{\mathcal{R}_{0}}=2.53\right.$, dashed line), the selection gradient at the maximum virulence $(\alpha=1)$ is positive below the dashed line (red area). When the basic reproduction number is set at its lower bound $\left(\widehat{\mathcal{R}_{0}}=1.26\right.$, plain line), the selection gradient is also positive for a range of virulence higher than one half $(\alpha \geq 50 \%)$ in the pink area. It is negative for lower virulences $(\alpha<50 \%)$ above the dashed line (green area). The unsafe burial proportion and the trade-off exponent are low $(\theta=0$ and $p=0.1)$. See Supplementary Material E for more details. 

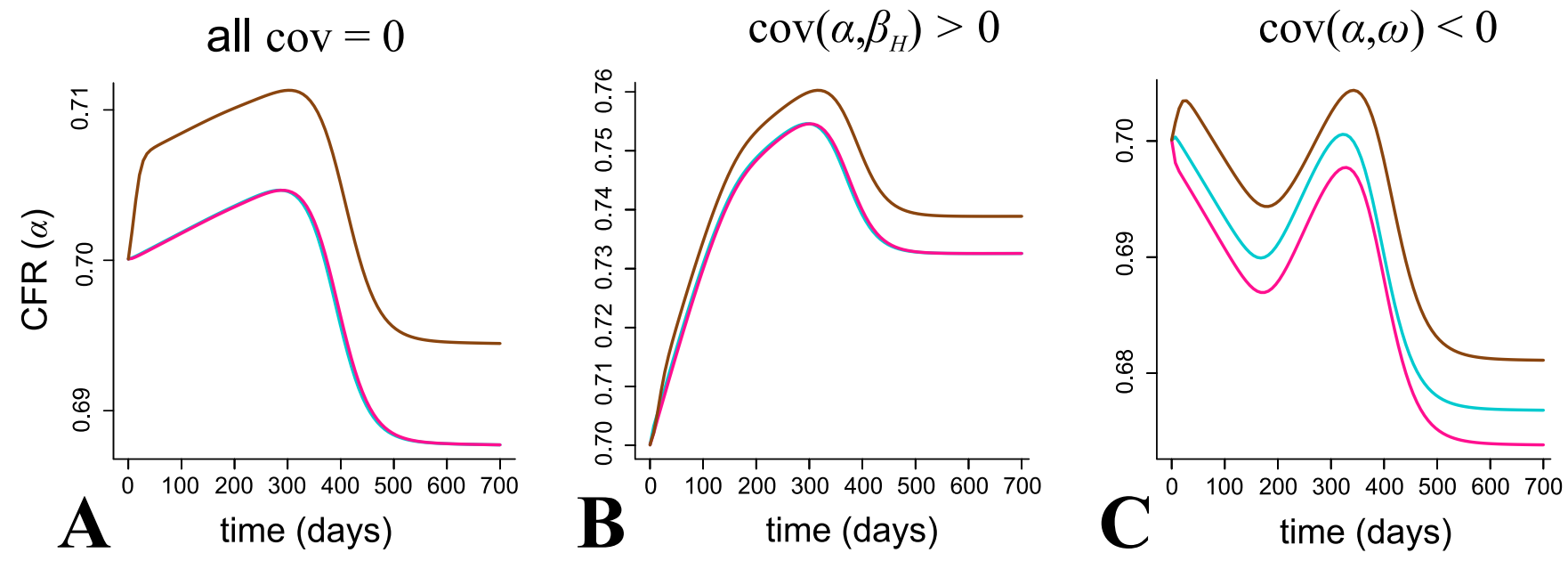

Figure 5: Short-term evolution of CFR with standing genetic variation in three scenarios.

A) Without correlations between traits, B) with a positive correlation between CRF and transmission rate and $\mathrm{C}$ ) with a negative correlation between $\mathrm{CRF}$ and latency period. The CFR averaged over the exposed individuals $\left(\bar{\alpha}^{E}\right)$ is depicted in cyan, over the symptomatic individuals $\left(\bar{\alpha}^{I}\right)$ in pink and over the infectious dead bodies $\left(\bar{\alpha}^{D}\right)$ in brown. Parameter values are shown in Table 1. See Supplementary Material G for details about the simulations. 